\title{
Using the Perceptron Algorithm to Find Consistent Hypotheses
}

\author{
Martin Anthony \\ Department of Statistical and Mathematical Sciences \\ London School of Economics, \\ Houghton Street, London WC2A 2AE, UK. \\ m.anthony@lse.ac.uk. \\ John Shawe-Taylor \\ Department of Computer Science \\ Royal Holloway and Bedford New College \\ Egham Hill, Egham, Surrey TW20 0EX, UK. \\ john@dcs.rhbnc.ac.uk.
}

\begin{abstract}
The perceptron learning algorithm yields quite naturally an algorithm for finding a linearly separable boolean function consistent with a sample of such a function. Using the idea of a specifying sample, we give a simple proof that this algorithm is not efficient, in general.
\end{abstract}

A boolean function $t$ defined on $\{0,1\}^{n}$ is linearly separable if there are $\alpha \in \mathbf{R}^{n}$ and $\theta \in \mathbf{R}$ such that

$$
t(x)= \begin{cases}1 & \text { if }\langle\alpha, x\rangle \geq \theta \\ 0 & \text { if }\langle\alpha, x\rangle<\theta\end{cases}
$$

where $\langle\alpha, x\rangle$ is the standard inner product of $\alpha$ and $x$. Given such $\alpha$ and $\theta$, we say that $t$ is represented by $[\alpha, \theta]$ and we write $t \leftarrow[\alpha, \theta]$. The vector $\alpha$ is known as the weight-vector, and $\theta$ is known as the threshold. This class of functions is the set of functions computable by the simple boolean perceptron (see $[8,9,6]$ ), and we shall denote it by $B P_{n}$. 
We now give a fleeting description of the perceptron learning algorithm, and refer to $[6,1]$ for more details. For any learning constant $\nu>0$, we have the perceptron learning algorithm $L_{\nu}$, devised by Rosenblatt [8, 9], which acts sequentially as follows. Let $t$ be any function in $B P_{n}$, which may be thought of as the target. The algorithm $L_{\nu}$ maintains at each stage a current hypothesis, which is updated on the basis of an example in $\{0,1\}^{n}$, presented together with its classification $t(x)$. (The initial hypothesis is some fixed 'simple' hypothesis. We shall take the initial hypothesis to have the all-0 vector as weight-vector, and threshold 0.) Suppose the current hypothesis is $h \leftarrow[\alpha, \theta]$ and that an example $x$ is presented. Then the new hypothesis is $h^{\prime} \leftarrow\left[\alpha^{\prime}, \theta^{\prime}\right]$ where

$$
\alpha^{\prime}=\alpha+\nu(t(x)-h(x)) x, \quad \theta^{\prime}=\theta-\nu(t(x)-h(x)) .
$$

The Perceptron Convergence Theorem [8,6] asserts that no matter how many examples are presented, the algorithm makes only a finite number of changes, or updates (provided $\nu$, which can be a function of $n$, is small enough).

As indicated in [3], given $t \in B P_{n}$ and a sample $\mathbf{x}=\left(x_{1}, x_{2}, \ldots, x_{m}\right)$ of examples, we may use $L_{\nu}$ to find a linearly separable boolean function which agrees with $t$ on $\mathbf{x}$ - that is, which is consistent with $t$ on $\mathbf{x}$. We simply keep cycling through $x_{1}$ to $x_{m}$ in turn, until no updates are made in a complete cycle. Thus, the perceptron algorithm (for any learning constant $\nu$ ) can be used as a consistent-hypothesis-finder (using terminology from [3]). A natural question is whether this is an efficient means of finding a consistent function. In fact, it is not, in the sense that the number of complete cycles required can be exponential in $m$, the size of the sample. This result appears to be accepted, but we have been unable to find a proof of it in the literature. We note that this is a very different result from those presented by Minsky and Papert[6] and Hampson and Volper [4] in their studies of the perceptron learning algorithm. Their results show that when the perceptron learning algorithm is used as an exact learning algorithm, the running time can be exponential in $n$, the domain dimension. Our result shows that, for fixed $n$, the running time of the related consistent-hypothesis-finder can be exponential in $m$, the number of examples presented. We remark that there is a polynomial time consistent-hypothesis-finder for $B P_{n}$ : rephrase the problem as a linear programme and use Karmarkar's algorithm (see [3]). Thus the problem of finding a consistent hypothesis has no intrinsic difficulty.

We shall consider the boolean function $f_{2 n}$ of $2 n$ variables with formula

$$
f_{2 n}=u_{2 n} \wedge\left(u _ { 2 n - 1 } \vee \left(u_{2 n-2} \wedge\left(u_{2 n-3} \vee\left(\ldots\left(u_{2} \wedge u_{1}\right)\right) \ldots\right),\right.\right.
$$

in the standard notation for describing boolean functions in terms of the literals $u_{1}, u_{2}$, the OR connective $\vee$ and the AND connective $\wedge$. This function, discussed 
in $[7,4,5]$, is in $B P_{n}$. (Indeed, all such 'nested' functions are; see [2].) The following easily obtained result is along the lines of results due to Muroga [7].

Proposition 1 Let $n$ be any positive integer and suppose $f_{2 n} \leftarrow[\alpha, \theta]$. Then $\alpha_{2 n} \geq$ $\sqrt{3}^{n-1} \min \left(\alpha_{1}, \alpha_{2}\right)$.

We have the following result, a special case of a more general 'specification' result from $[2]$.

Proposition 2 Let the set $S_{n} \subseteq\{0,1\}^{2 n}$ of cardinality $2 n+1$ be defined for each positive integer $n$ as follows. $S_{1}=\{(0,1),(1,0),(1,1)\}$, and, for $n \geq 1$,

$$
S_{n+1}=\left\{x 01: x \in S_{n}\right\} \cup\{(11 \ldots 10),(00 \ldots 011)\} .
$$

Then the only function $h \in B P_{n}$ consistent with $f_{2 n}$ on $S_{n}$ is $f_{2 n}$ itself.

Combining these two results, we obtain the result we seek.

Theorem 3 For any fixed $\nu>0$, the consistent-hypothesis-finder arising from the perceptron learning algorithm $L_{\nu}$ does not always run in time polynomial in the size of its input.

Proof: Suppose we take the target $t$ to be $f_{2 n}$ and we take $S_{n}$ as the input to the consistent-hypothesis-finder. Suppose the initial hypothesis is $h \leftarrow[(00, \ldots, 0), 0]$. Let $N$ be the number of updates made before a consistent hypothesis is produced. By Proposition 2, this consistent hypothesis must be $f_{2 n}$ itself, and so if it is represented by $[\alpha, \theta]$, then $\alpha_{1}, \alpha_{2}>0$ and, by Proposition $1, \alpha_{2 n} \geq \sqrt{3}^{n-1} \min \left(\alpha_{1}, \alpha_{2}\right)$. After $N$ updates, the maximum entry in the new weight-vector $\alpha^{\prime}$ is at most $N \nu$ and the minimum entry is certainly at least $\nu$. Hence the ratio of maximum entry to minimum entry is at most $N$. But, since the final output weight-vector has this ratio at least equal to $\alpha_{2 n} / \min \left(\alpha_{1}, \alpha_{2}\right)>\sqrt{3}^{n-1}$, it follows that $N \geq \sqrt{3}^{n-1}$, which is exponential in $n$, and hence in $2 n+1$, the size of the input.

This result also holds if $\nu=\nu(n)$ is a function of $n$, bounded above by some constant. (Usually, this is certainly the case since $\nu$ is taken to be decreasing with $n$.) 


\section{References}

[1] M. Anthony and N. Biggs, Computational Learning Theory: An Introduction, Cambridge University Press: Cambridge, UK, 1992.

[2] M. Anthony, G. Brightwell, D. Cohen and J. Shawe-Taylor, On exact specification by examples, in COLT'92, Proceedings of the Fifth Annual Workshop on Computational Learning Theory, July 1992.

[3] A. Blumer, A. Ehrenfeucht, D. Haussler and M. Warmuth, Learnability and the Vapnik-Chervonenkis Dimension. Journal of the ACM, 36(4), 1989: 929965 .

[4] S.E. Hampson and D.J. Volper, Linear function neurons: structure and training. Biological Cybernetics 53, 1986: 203-217.

[5] N. Littlestone, Learning quickly when irrelevant attributes abound: a new linear threshold learning algorithm. Machine Learning, 2(4), 1988: 285-318.

[6] M. Minsky and S. Papert, Perceptrons. MIT Press, Cambridge, MA., 1969. (Expanded edition 1988.)

[7] S. Muroga, Lower bounds of the number of threshold functions and a maximum weight. IEEE Transactions on Electronic Computers, 14, 1965: 136-148.

[8] F. Rosenblatt, Two theorems of statistical separability in the perceptron. In Mechanisation of Thought Processes: Proceedings of a Symposium Held at the National Physical Laboratory, November 1958. Vol. 1. HM Stationery Office, London, 1959.

[9] F. Rosenblatt, Principles of Neurodynamics. Spartan, New York, 1962. 